\title{
Impulsively generated oscillations in a 3D coronal loop
}

\author{
D. J. Pascoe ${ }^{1}$, I. De Moortel ${ }^{1}$, and J. A. McLaughlin ${ }^{1}$
}

\author{
School of Mathematics and Statistics, University of St Andrews, St Andrews, KY16 9SS, UK \\ e-mail: david.pascoe@st-andrews.ac.uk
}

Received 3 April 2009 / Accepted 7 July 2009

\begin{abstract}
Aims. The effect of changing the attack angle for the interaction of a fast MHD wave with a 3D coronal loop is studied, to investigate to what extent the properties of the excited transverse kink mode oscillations of the loop depend on this angle.

Methods. 3D numerical simulations are performed of the interaction of a fast MHD wave, generated by a pressure pulse, with a 3D coronal loop. The loop itself is modelled as a density enhancement (with a finite plasma beta) within a magnetic arcade. The initial pressure pulse has a width comparable to the loop diameter and is situated outside of the loop, at the same height as the loop apex. This height is kept fixed but the (horizontal) angle between the pressure pulse and the loop is varied.

Results. We find that the global, transverse kink mode is efficiently excited for a range of attack angles and qualitatively in agreement with theoretical expectations. The period and damping time are found to be independent of the attack angle. For larger values of the attack angle, the global (longitudinal) slow wave is excited, whereas for intermediate values the second harmonic kink mode is also present.
\end{abstract}

Key words. Sun: corona - Sun: oscillations - magnetohydrodynamics (MHD)

\section{Introduction}

Flares and coronal mass ejections (CMEs) in the solar atmosphere can impulsively excite magnetic loop oscillations. Such coronal oscillations have now been observed with several solar instruments, including the TRACE satellite (e.g. Nakariakov et al. 1999; Aschwanden et al. 1999, 2002; Wang \& Solanki 2004) and Hinode (Ofman \& Wang 2008). Furthermore, these oscillations have been successfully interpreted as MHD wave motions (e.g. Edwin \& Roberts 1983; Roberts 2004; De Moortel 2005; Nakariakov \& Verwichte 2005). Thus, coronal seismology has become a viable diagnostic technique, i.e. with realistic modelling, we can indirectly measure the structure and physical properties of the medium through which these MHD waves travel.

Coronal seismology was first suggested by Uchida (1970) and later discussed by Roberts et al. (1984). Nakariakov \& Ofman (2001) used TRACE observations to demonstrate that coronal loop oscillations can be used to determine the magnetic field of an oscillating loop. More recently, Van Doorsselaere et al. (2008) have made magnetic field measurements using loop oscillations observed with Hinode/EIS. Comprehensive reviews of coronal seismology can be found in De Moortel (2005), Nakariakov \& Verwichte (2005) and Banerjee et al. (2007). Coronal seismology has also been developed extensively through several theoretical studies (including Ofman 2007; Selwa et al. 2007a; Taroyan et al. 2007; Van Doorsselaere et al. 2007; Wang et al. 2007; Ofman \& Wang 2008), in particular including the effects of curvature (Van Doorsselaere et al. 2004; Gruszecki et al. 2007), longitudinal density variations (Erdelyi \& Verth 2007; Verth et al. 2007; Pascoe et al. 2009) and transverse structuring (Arregui et al. 2007; Ballai 2007; Pascoe et al. 2007).

A key result found in these loop observations is that the oscillations decay rapidly within a few periods. Several proposed theories have been put forward to explain this strong damping, including enhanced viscosity (Nakariakov et al. 1999), wave leakage (Brady \& Arber 2005; Terradas et al. 2005a), phase mixing with enhanced resistivity (Ofman \& Aschwanden 2002) and resonant absorption (Ruderman \& Roberts 2002). Reviews of the different damping mechanisms can be found in, for example, Roberts (2004). Another unexplained property is that only a limited number of loops are seen to oscillate under the same impulsive excitation, i.e. there is a selectivity to the excitations. It is most likely that a detailed and numerical treatment of a realistic coronal loop, together with high-resolution and spectral observations, is required to gain further understanding of these issues (Roberts 2000).

The aim of this paper is to impulsively excite MHD waves in a three-dimensional loop model, and in particular investigate the importance of the attack angle in determining the efficiency of such excitations. Several authors have investigated MHD wave excitation in 2D geometries (e.g. Selwa et al. 2005, 2006, 2007a,b; Gruszecki et al. 2006, 2008; Del Zanna et al. 2005). However, our investigation is only possible in a fully $3 \mathrm{D}$ model, and thus this paper represents the first such investigation of the importance of attack angle in a 3D geometry. Other studies of 3D MHD models of wave activity in coronal active regions were performed by Ofman \& Thompson (2002), Terradas \& Ofman (2004), Miyagoshi et al. (2004) and Ofman (2009). Ofman $(2005,2007)$ demonstrated the potential of such models: that the analysis of such three-dimensional wave propagation can serve as a diagnostic of active region parameters. McLaughlin \& Ofman (2008) extended these studies with the inclusion of an individual loop density structure. This creates a density contrast between the loop and the ambient plasma, and this can support trapped MHD modes (Roberts et al. 1983), as well as phase mixing (Heyvaerts \& Priest 1983) and resonant absorption (e.g. Ruderman \& Roberts 2002 and references therein) in the loops. 


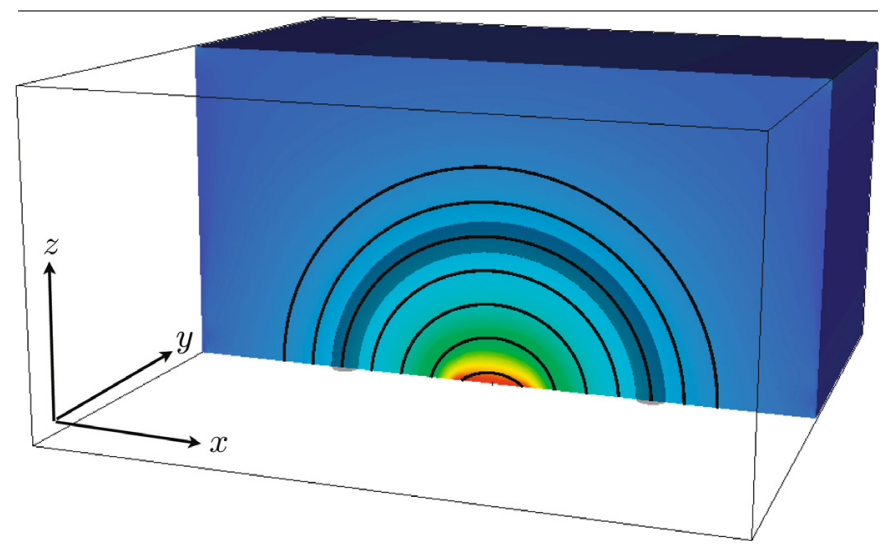

Fig. 1. 3D Numerical domain showing the density-loaded model coronal loop (shaded region) as well as a few representative magnetic field lines outlining the arcade structure. The colour gradient in the background represents the magnetic field strength.

Finally, Terradas et al. (2006b) studied kink modes of oscillation in a curved coronal loop using a 2D linear, zero $\beta$, toroidal model with a power-law density profile. By considering linearised perturbation about this equilibrium, two types of fundamental kink modes were found with either (mainly) horizontal or vertical polarisations. It was also noted that the oscillations were damped and that this was due to resonant absorption and wave leakage, with the former being the dominant damping mechanism.

Wang et al. (2008) considered the observational signatures of horizontal and vertical kink modes in their fundamental mode and second harmonic and found that, for many combinations of viewing angle and loop geometry, it can be difficult to distinguish between different types of kink mode using only a time series of images.

The paper is organised as follows: the method of generating an equilibrium state is described in Sect. 2. In Sect. 3 the results are presented and Sect. 4 contains the discussion. Conclusions are given in Sect. 5.

\section{Model and equilibrium setup}

The aim of our study is to examine the behaviour of oscillating loops in a 3D coronal environment. In this first paper, we consider a coronal loop which is part of an arcade structure. Figure 1 shows the coronal loop as well as a few representative magnetic field lines in the 3D numerical domain. The magnetic field is a 2 D arcade with $B \sim 1 / r$ (e.g. Brady et al. 2006) and a loop of (minor) radius $a$ within this arcade is modelled as a density enhancement. The chosen magnetic field profile becomes singular as $r \rightarrow 0$ and so is not used for small $r$ where gas pressure alone is used. To ensure that this deviation from equilibrium does not affect our subsequent results, the simulations were initially run without a driver to ensure that any flows arising from this (local) non-equilibrium are small. The profile of the density enhancement forming the 3D coronal loop is a modification of the Epstein profile (e.g. Nakariakov \& Roberts 1995) and is given by:

$\rho(x, y, z)=\rho_{\mathrm{e}}+\left(\rho_{0}-\rho_{\mathrm{e}}\right) \operatorname{sech}^{2}\left(\frac{\left|r-r_{\mathrm{c}}\right|}{a}\right)^{q} \operatorname{sech}^{2}\left(\frac{|y|}{a}\right)^{q}$

where $\rho_{0}$ is the internal density, $\rho_{\mathrm{e}}$ is the external density, $r=$ $\sqrt{x^{2}+z^{2}}, r_{\mathrm{c}}$ is the major loop radius and $q$ is a steepness parameter. The cartoons in Fig. 2 show a side and top view of the a)

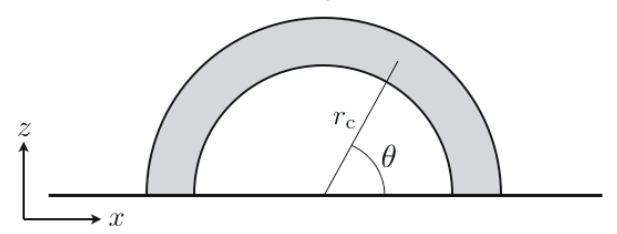

b)

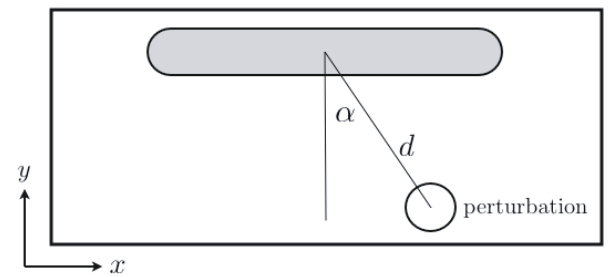

Fig. 2. Cartoon showing a view of the coronal loop a) from the side and b) from the top. The pressure pulse is located a constant distance $d$ away from the apex, at a height equal to the loop major radius $h=r_{\mathrm{c}}$. The position of the pulse is determined by an attack angle $\alpha$. The loop has a minor radius $a$ and the angle $\theta$ describes the position along the loop axis.

modelled coronal loop and illustrate the definition of the angles $\theta$ (along the loop) and $\alpha$ (between the loop apex and the pressure pulse). In our simulations we use a density contrast ratio of $\rho_{0} / \rho_{\mathrm{e}}=10$ based on the value used by Nakariakov \& Ofman (2001). Figure 3 shows the density profiles corresponding to the symmetric Epstein profile defined above (solid line, $q=1$ ) and the step profile which can be modelled by $q \rightarrow \infty$ (dashed line) and which we will use as a comparison. The temperature profile is defined to ensure thermal pressure balance.

The simulations are performed using the MHD code LARE3D (Arber et al. 2001) to solve the (nonlinear) 3D MHD equations:

$$
\begin{aligned}
\rho\left[\frac{\partial \boldsymbol{v}}{\partial t}+(\boldsymbol{v} \cdot \nabla) \boldsymbol{v}\right] & =-\nabla p+\left(\frac{1}{\mu} \nabla \times \boldsymbol{B}\right) \times \boldsymbol{B}, \\
\frac{\partial \boldsymbol{B}}{\partial t} & =\nabla \times(\boldsymbol{v} \times \boldsymbol{B})+\eta \nabla^{2} \boldsymbol{B}, \\
\frac{\partial \rho}{\partial t}+\nabla \cdot(\rho \boldsymbol{v}) & =0, \\
\rho\left[\frac{\partial \epsilon}{\partial t}+(\boldsymbol{v} \cdot \nabla) \epsilon\right] & =-p \nabla \cdot \boldsymbol{v}+\frac{1}{\sigma}|\boldsymbol{j}|^{2}
\end{aligned}
$$

where $\rho$ is the mass density, $\boldsymbol{v}$ is the plasma velocity, $\boldsymbol{B}$ the magnetic induction (usually called the magnetic field), $p$ is the plasma pressure, $\mu=4 \pi \times 10^{-7} \mathrm{Hm}^{-1}$ is the magnetic permeability, $\sigma$ is the electrical conductivity, $\eta=1 / \mu \sigma$ is the magnetic diffusivity, $\epsilon=p / \rho(\gamma-1)$ is the specific internal energy density, where $\gamma=5 / 3$ is the ratio of specific heats, and $\boldsymbol{j}=\nabla \times \boldsymbol{B} / \mu$ is the electric current density. The boundary conditions used are line-tied (zero velocity, zero gradient for other variables) at the lower boundary $(z=0)$ to simulate reflective loop footpoints. Elsewhere, the open corona is approximated using damping layers to avoid reflection of any perturbations back into the numerical domain. Numerical tests were carried out to confirm that these damping layers do not affect the subsequent dynamical evolution inside the computational domain. The numerical domain is about $180 \times 180 \times 90 \mathrm{Mm}$ with a resolution of $200 \times 200 \times 100$ gridpoints. Numerical results were also checked by doubling the numerical resolution. In this study, we model a 


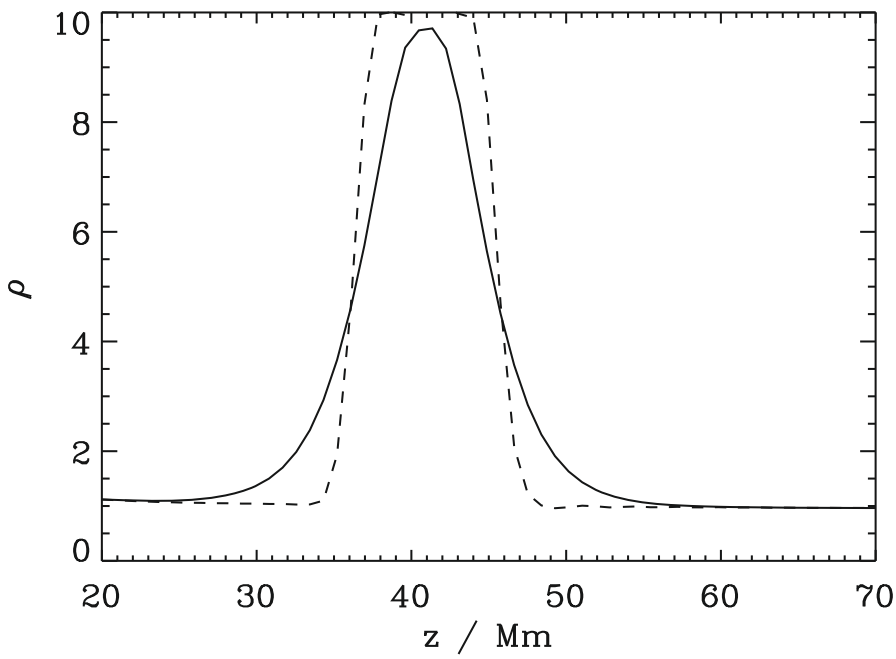

Fig. 3. Density profiles corresponding to the symmetric Epstein profile defined in Eq. (1) (solid line, $q=1$ ) and step profile (dashed line, $q \rightarrow$ $\infty)$.

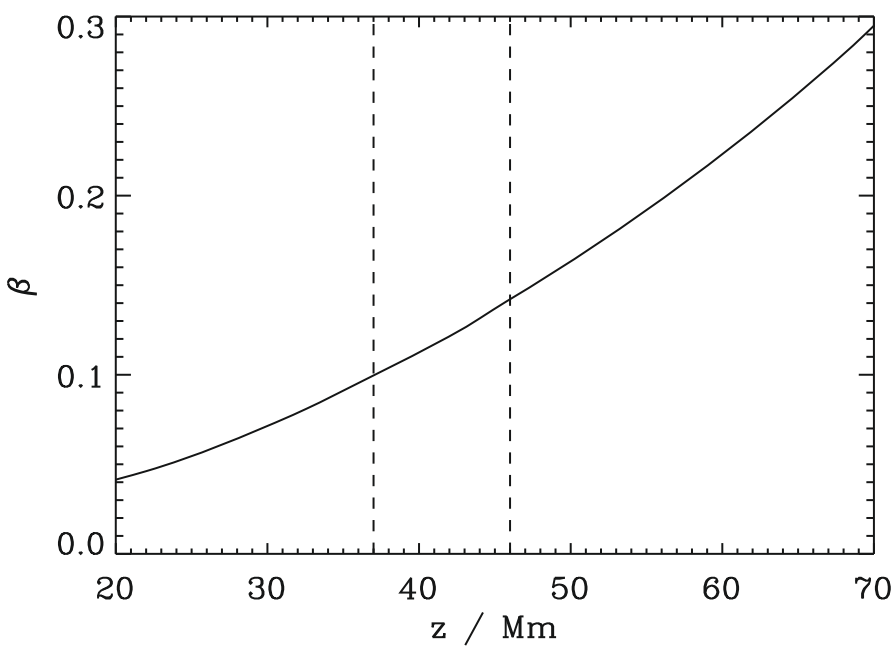

Fig. 4. Plasma $\beta$ as a function of radial distance. A finite value of $\beta=$ 0.1 is used to avoid density enhancement at the loop apex driven by the ponderomotive force. The dashed lines outline the location of the coronal loop.

loop of length $L=\pi r_{\mathrm{c}}=130 \mathrm{Mm}$, based on the length of the oscillating loop analysed by Nakariakov et al. (1999), and a diameter $2 a=9 \mathrm{Mm}$. This diameter is about 4 times larger than the observed diameter $(\approx 2 \pm 0.36 \mathrm{Mm})$ due to numerical restrictions. Velocities are normalised to give a kink speed of about $1 \mathrm{Mm} \mathrm{s}^{-1}$ at the loop apex, consistent with the kink speed estimated by Nakariakov et al. (1999). We consider the medium to be ideal and hence set $\eta=0$.

In our simulations we use a finite value for the plasma $\beta$ (the ratio of thermal and magnetic pressures) to avoid ponderomotive effects (Terradas \& Ofman 2004). Figure 4 shows the plasma $\beta$ as a function of radial distance $r ; \beta$ increases with $r$ since the magnitude of the magnetic field, and hence the magnetic pressure $p_{\text {mag }}=B^{2} / 2 \mu$, decreases as a function of radial distance. The temperature is chosen such that $\beta=0.1$ at the loop apex. Tests showed this value to be sufficiently large to counteract ponderomotive-driven longitudinal flows and avoid the resulting density enhancement at the loop apex.

\subsection{Estimation of required pressure pulse}

Although it is clear that the observed, transverse coronal loop oscillations are excited impulsively as a consequence of a nearby explosive event, the exact nature of the excitation mechanism is unclear. In this study, we choose to excite the loop oscillations with a nearby pressure pulse. In this respect, our setup differs from Miyagoshi et al. (2004) (who also consider a loop embedded in an arcade) who trigger the oscillation in situ with a velocity perturbation at the loop apex.

To determine the size of the pressure pulse required to generate realistic loop oscillations, we can use an order of magnitude estimate. From Newton's second law we have that

$M \frac{V_{0}}{\delta t} \approx \delta p A$,

where $M$ is the mass of the loop, $V_{0}$ is the velocity of the loop, and $\delta t$ is the time that the pressure pulse, $\delta p$, is applied to the area, $A$, of the loop. For a cylindrical loop of length $L$ and radius $a$, the mass is $M=\rho_{0} L \pi a^{2}$, where $\rho_{0}$ is the density inside the loop. The area of loop exposed is $A=2 L_{*} a$, where $L_{*}$ is the length hit by the pulse, giving

$\frac{\delta p}{p} \approx \frac{V_{0}}{\delta t} \frac{\rho_{0}}{2} \frac{L}{L_{*}} \frac{\pi a}{p}$.

Introducing the external sound speed $C_{\mathrm{s}}=\sqrt{\gamma p / \rho_{\mathrm{e}}}$, this equation can be rearranged to give

$\frac{\delta p}{p} \approx \frac{\gamma V_{0}}{C_{\mathrm{s}}^{2}} \frac{a}{\delta t} \frac{\pi}{2} \frac{\rho_{0}}{\rho_{\mathrm{e}}} \frac{L}{L_{*}}$.

As an illustration, taking $\rho_{0} / \rho_{\mathrm{e}}=10, L / L_{*} \approx 2, \delta t=5 \mathrm{~s}, \gamma=$ $5 / 3, C_{\mathrm{s}}=0.3 \mathrm{Mm} \mathrm{s}^{-1}, a=4.5 \mathrm{Mm}$ and $V_{0}=0.2 \mathrm{Mm} \mathrm{s}^{-1}$ we obtain $\delta p / p \approx 100$ at the loop itself, requiring $\delta p / p \approx 1000$ at the location of a pulse of size approximately $10 \mathrm{Mm}$, located about $40 \mathrm{Mm}$ away.

\section{Excitation of loop oscillations}

The simulations are initially run for a number of timesteps without a driver to confirm that any flows resulting from the nonequilibrium near $r=0$ are negligibly small. Subsequently, a pressure pulse is applied inside the numerical domain (but outside the coronal loop). This perturbation is modelled as a Gaussian pressure enhancement (1000 times the background value), located at the same height as the loop apex but at a distance of $d \approx 40 \mathrm{Mm}$ away (see calculation above). The position in the $x y$-plane is defined by an attack angle $\alpha$ (see Fig. 2). The width of $10 \mathrm{Mm}$ of the pressure pulse determined above is comparable to the loop diameter. By placing the pulse at the same height as the loop apex we aim to predominantly excite horizontal kink oscillations rather than vertical oscillations studied elsewhere (see Sect. 1).

After the pressure enhancement is applied, it travels through the numerical domain as a fast magneto-acoustic wave. The interaction of the wave with our coronal loop leads to impulsively excited oscillations in the loop, which we will discuss in more detail below. Once the fast magneto-acoustic wave generated by the pressure pulse reaches the boundaries of the numerical box, the damping layers ensure that no perturbation is reflected back into the domain.

We repeat our simulations for a range of different values of $\alpha$ to investigate the effect of the attack angle upon the resulting loop oscillations. We study the nature and amplitude of the oscillations, the dominant frequency and the signal quality (ratio of decay time and period). 
a)

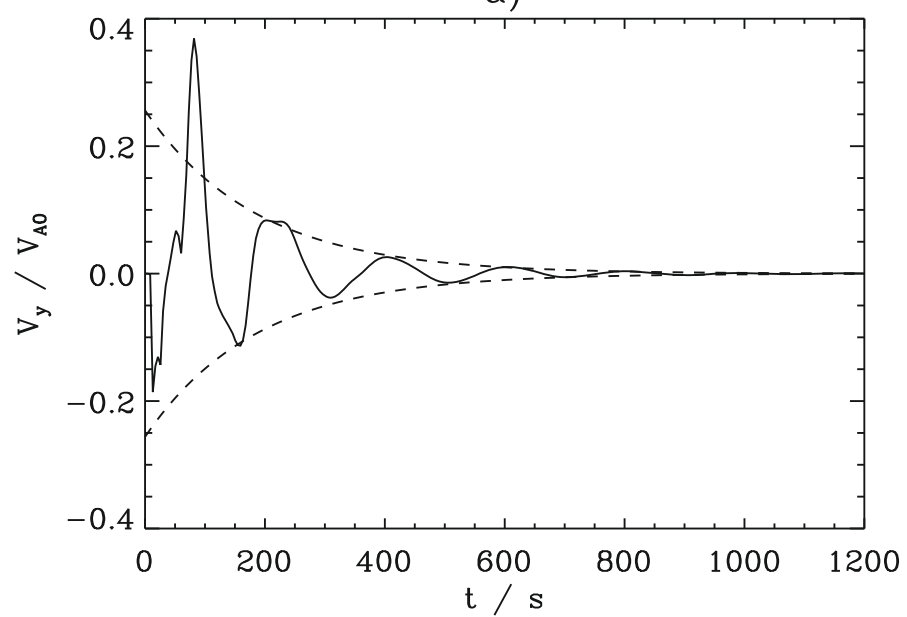

b)

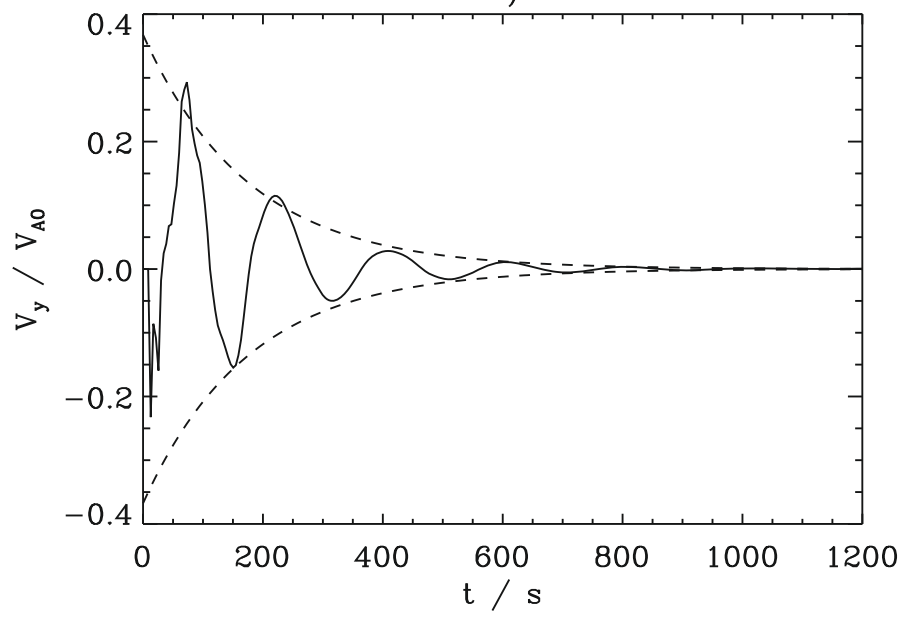

Fig. 5. Transverse velocity signal $v_{y}$ (normalised to the Alfvén speed at the loop apex) measured at the loop apex for an attack angle of a) 0 degrees and b) 30 degrees. The dashed lines show fits to an exponential decay.

\subsection{Kink mode}

For small values of the attack angle (0-30 degrees), the loop plasma is mainly perturbed in the $y$-direction, corresponding to the transverse kink mode. Figure 5 shows the (normalised) transverse velocity signal $v_{y}$, measured at the loop apex for an attack angle of 0 degrees and 30 degrees. We can see that in both cases, the signal has two phases; an initial, aperiodic, transitory phase during which the fast MHD wave interacts with the loop, and a second phase in which the MHD mode is excited. This second phase may be approximated as a damped harmonic oscillation. The dashed line envelopes shown in both figures correspond to an exponential damping profile fitted to the latter (periodic) part of the $v_{y}$ oscillations. The transient behaviour between the initial and the periodic phase was studied for a cylindrical loop by Terradas et al. (2007a) in terms of radiating "trig" leaky modes (see also Cally 1986, 2003).

Figure 6 shows the periodogram of the transverse velocity signal shown in Fig. 5(a). The spectral amplitude shows a single peak frequency corresponding to the kink mode. The peak is rather broad, due to a combination of the quasi-periodic nature of the velocity signal, as well as its relatively short duration. The maximum of this peak corresponds to a period of oscillation of $165 \mathrm{~s}$. Wavelet analysis confirmed that this period remains

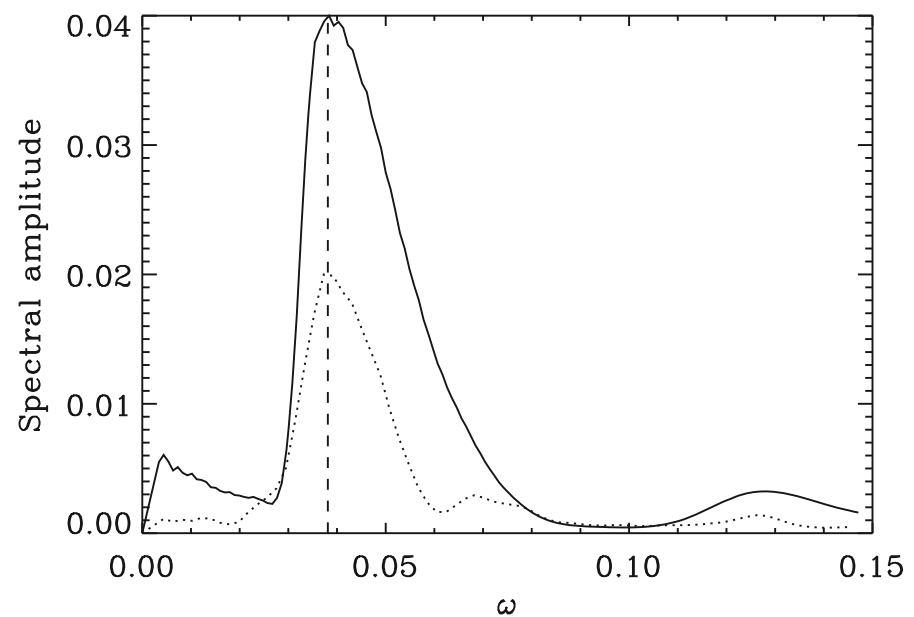

Fig. 6. Periodogram of the transverse velocity signal measured at the loop apex (solid line) for an attack angle of 0 degrees (see Fig. 5a). The dotted line (normalised for clarity) corresponds to the velocity signal located outside the loop at $y=4 a$.

roughly constant for the duration of the simulation. The dotted line in Fig. 6 corresponds to the velocity signal located outside the loop at $y=4 a$. It has been normalised to half the amplitude of the solid line for clarity, and it shows that the kink mode eigenfunction has tails that extend into the external medium.

In the long-wavelength limit $(k a \rightarrow 0)$, the period of the global kink mode is given by

$P_{\text {kink }}=\frac{2 L}{C_{\mathrm{k}}}$,

where the kink speed is

$C_{\mathrm{k}}=\left(\frac{\rho_{0} C_{\mathrm{A} 0}^{2}+\rho_{\mathrm{e}} C_{\mathrm{Ae}}^{2}}{\rho_{0}+\rho_{\mathrm{e}}}\right)^{1 / 2}$.

Normally, the kink speed for a cylindrical loop is closer to the Alfvén speed inside the loop (Nakariakov 2007). In our simulations we have $k a=0.1$ and a kink speed $C_{\mathrm{k}}=1.0 \mathrm{Mm} \mathrm{s}^{-1}$ for $C_{\mathrm{A} 0}=0.8 \mathrm{Mm} \mathrm{s}^{-1}$ and $C_{\mathrm{Ae}} \approx 2.0 \mathrm{Mm} \mathrm{s}^{-1}$. (Note that the external Alfvén speed varies due to the magnetic field profile.) With a loop length of $130 \mathrm{Mm}$, Eq. (5) gives a kink mode period of about $260 \mathrm{~s}$. However, the period of oscillation obtained from our simulations (about $165 \mathrm{~s}$ for the Epstein profile and about $145 \mathrm{~s}$ for the step profile) is much shorter than the theoretically predicted period of $260 \mathrm{~s}$. Deriving a phase speed, using $L=130 \mathrm{Mm}$ and $P \approx 150 \mathrm{~s}$ we obtain $C_{\mathrm{p}}=2 L / P \approx 1.7 \mathrm{Mm} \mathrm{s}^{-1}$. Our phase speed is therefore higher than expected and although still less than, it is close to the external Alfvén speed. We will discuss this modification of the period further in Sect. 4.

If the excited transverse velocity signal is indeed the global kink mode for the loop, the amplitude of the oscillation is expected to be largest at the loop apex and zero at both footpoints. Figure 7 shows the spectral amplitude as a function of the angle around the loop $\theta$, where $\theta$ of 0 and 180 degrees correspond to the respective loop footpoints and the apex is at 90 degrees (see Fig. 2). The attack angle in this particular example is 0 degrees. The crosses correspond to the actual datapoints whereas the solid line is a smoothed fit to these points. It is clear that the amplitude of the oscillation is indeed zero at the footpoints and has a single maximum at the apex, corresponding to a global mode with a wavelength of twice the loop length. 


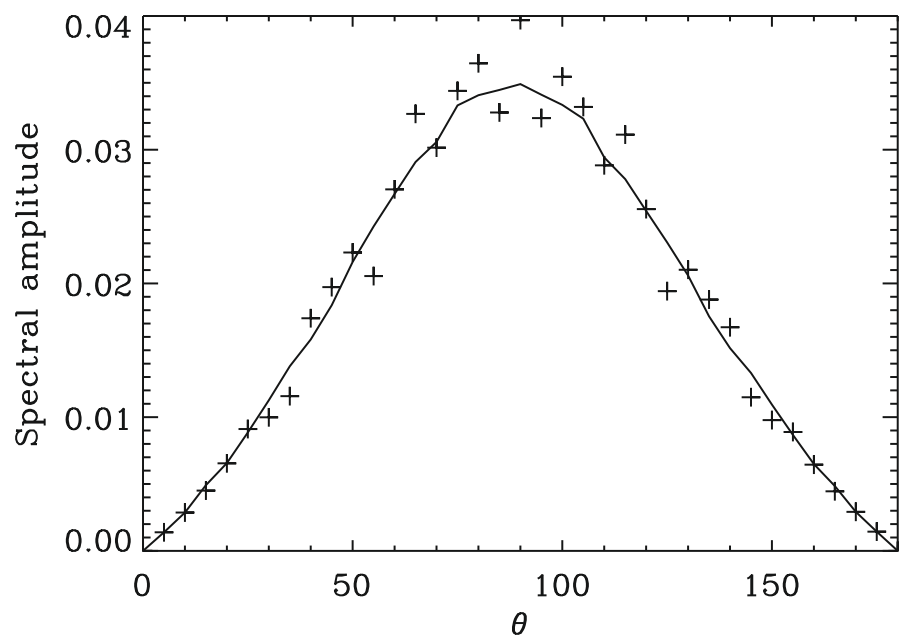

Fig. 7. The spectral amplitude of the kink mode as a function of the angle around the loop $\theta$. The data points are represented by crosses and the line is a smoothed fit. The attack angle $\alpha$ is 0 degrees.

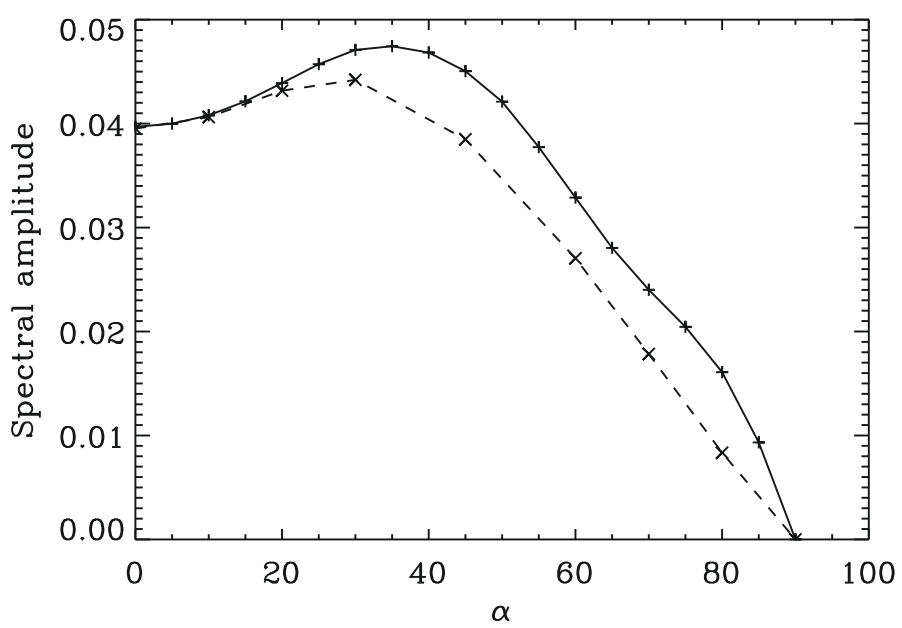

Fig. 8. The spectral amplitude of the global kink mode as a function of the attack angle $\alpha$. The solid and dashed lines correspond to the Epstein and step density profiles, respectively.

Let us now vary the attack angle $\alpha$; for a range of attack angles, we take the periodogram of the transverse velocity displacement at the loop apex and determine the amplitude of the peak in the periodogram. Figure 8 shows this maximum spectral amplitude, measured at the loop apex, as a function of the attack angle. For small values of attack angle, the transverse kink mode is excited efficiently, leading to relatively high spectral amplitudes. As expected, the excitation of the transverse perturbation becomes less efficient for larger values of the attack angle. For $\alpha=90$ degrees, the $v_{y}$ component is no longer excited at all. The small increase in spectral amplitude for medium values of $\alpha$ is most likely artificial. For $\alpha \approx 30$ degrees (see Fig. 5b), the signal at the loop apex resembles a harmonic oscillation rather quickly, whereas the signal for $\alpha=0$ degrees (see Fig. 5a) remains quasi-period for a slightly longer time. The combination of the relatively low signal quality (see below) and the "cleaner" transverse velocity for an attack angle of 30 degrees leads to a slightly higher peak in the corresponding periodogram. To show that this is indeed the case, the actual maximum amplitude of the transverse velocity perturbation at the loop apex is plotted in Fig. 9, again as a function of the attack angle. The largest amplitude perturbation does indeed occur for zero attack angle,

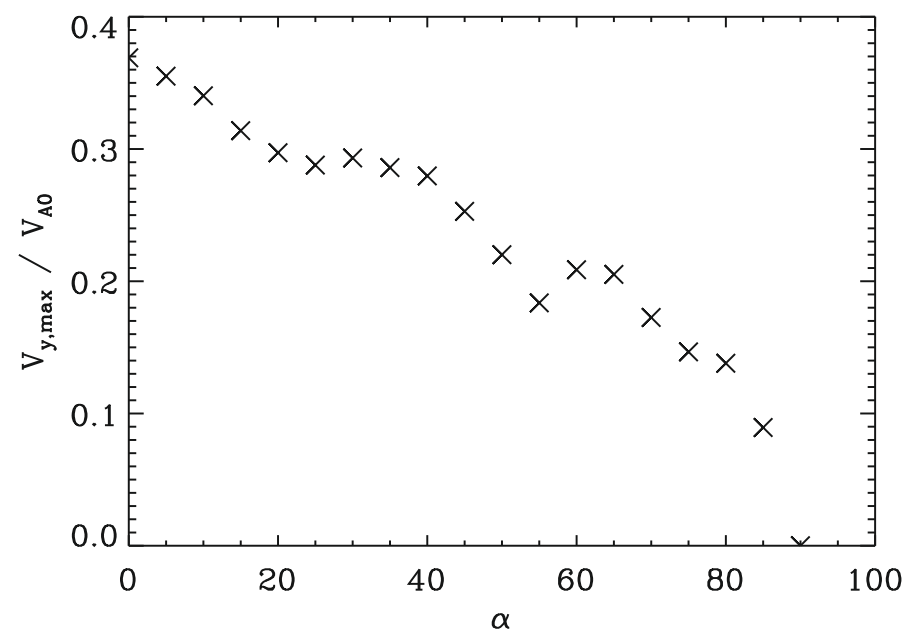

Fig. 9. The dependence of the maximum amplitude of the perturbation on the attack angle $\alpha$.

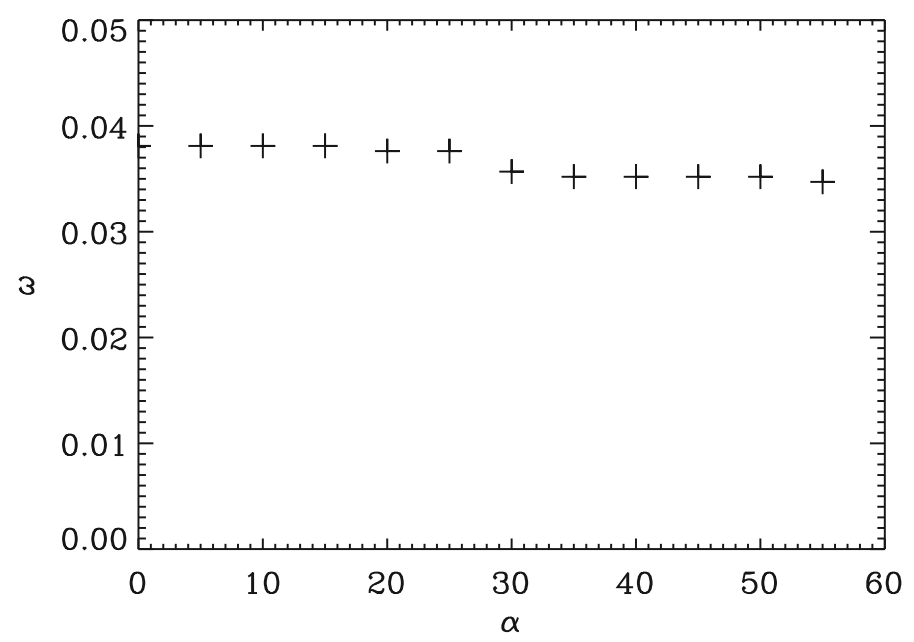

Fig. 10. The frequency of oscillation $\omega$ of the kink mode is independent of the attack angle $\alpha$.

with the transverse perturbation decreasing steadily for increasing values of $\alpha$. This confirms that the maximum in the spectral amplitude around $\alpha=30$ degrees is due to the larger peak in the periodogram and not due to an actual larger transverse perturbation.

So far, we have looked at both the amplitude of the transverse velocity displacement at the loop apex and the corresponding spectral amplitude derived from the periodogram. Figure 10 shows the (cyclic) frequency of oscillation as a function of the attack angle $\alpha$. The frequency of the global MHD kink mode of a coronal loop is determined entirely by the loop properties and plasma parameters such as the internal and external Alfvén speed. Hence, once it is properly established (i.e. after any initial aperiodic transitory phase) the frequency of this mode should be independent of the excitation mechanism. Figure 10 confirms this is indeed the case in our numerical simulations, with the frequency $\omega$ remaining roughly constant for all values of $\alpha$. The larger attack angles (tending towards 90 degrees) have been excluded from this graph, as the amplitude of the transverse velocity at the loop apex becomes very small and hence subject to increasingly larger errors.

The final parameter we investigate is the signal quality of the excited kink mode. We define the signal quality, $Q$ as the ratio of the decay time and the oscillation period $(Q=\tau / P)$. 


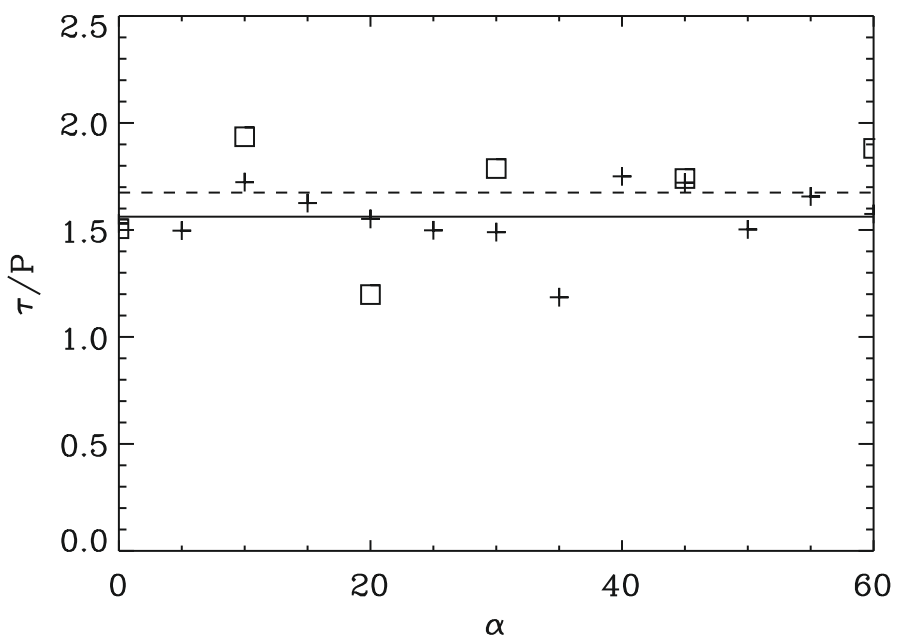

Fig. 11. Kink mode signal quality as a function of the attack angle. The solid and dashed lines represent the mean values for the Epstein and step density profiles, respectively.

For each value of the attack angle $\alpha$, the decay time is estimated by fitting the oscillations (see dashed lines in Fig. 5) to an exponential decay with decay constant $1 / \tau$. Figure 11 shows the kink mode signal quality as a function of the attack angle. The crosses correspond to the Epstein profile with $q=1$, whereas the squares represent the step profile $(q \rightarrow \infty)$. The solid and dashed lines represent the mean values for the Epstein and step density profiles, respectively. As the exponential decay is fitted to a small number of (decaying) peaks, the errors on the fit are relatively large, causing the spread in the individual data points. However, comparing with the mean value, the majority of the datapoints are relatively close and hence the signal quality seems largely independent of the attack angle. Although the mean value is slightly higher for the step profile (dashed line), the scatter on the individual data points is too large to attribute significance to this slight difference.

\subsection{Higher harmonics}

So far we have only considered the global or fundamental kink mode as this is the main focus of our study. De Moortel \& Brady (2007) report the case of a flare-induced transverse oscillation of a coronal loop observed by TRACE in which the fundamental mode appeared to be absent and the oscillation was predominantly that of the second harmonic. Multiple oscillation modes in loops of a post-flare arcade have also been observed by Verwichte et al. (2004).

The line-tied boundary conditions in our model are also satisfied for higher longitudinal harmonics i.e. $N>1$ where $\lambda=2 L / N$ or equivalently $k_{N}=N \pi / L$. The size and location of the pulse are the important parameters. We choose our driver to be comparable in size and distance to the loop minor and major radii, respectively. We would expect higher harmonics to become more important for the case of a perturbation that is closer to the loop and more localised.

The second harmonic kink mode has $N=2$ or $\lambda=L$. This mode has a node at the apex and so would not be seen in Figs. 5 and 6 even if present. Figure 12 shows the transverse velocity signal measured at the apex and at $\theta=45$ degrees for an attack angle of 45 degrees. There is no evidence for higher harmonics in the apex signal, but the signal at $\theta=45$ degrees (where we expect the second harmonic to have an anti-node) shows signs

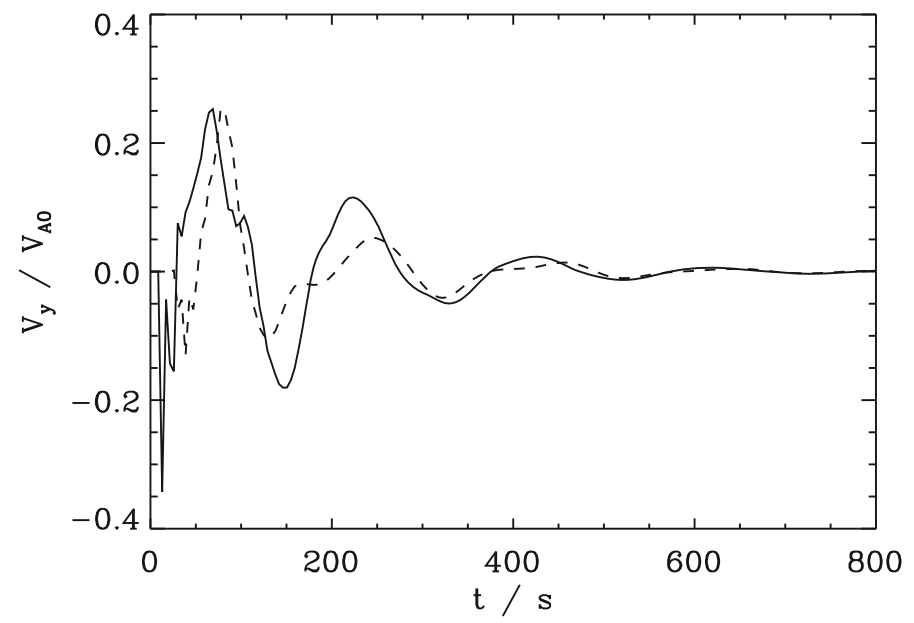

Fig. 12. Transverse velocity signal measured at the apex (solid line) and at $\theta=45$ degrees (dashed line) for an attack angle of 45 degrees.

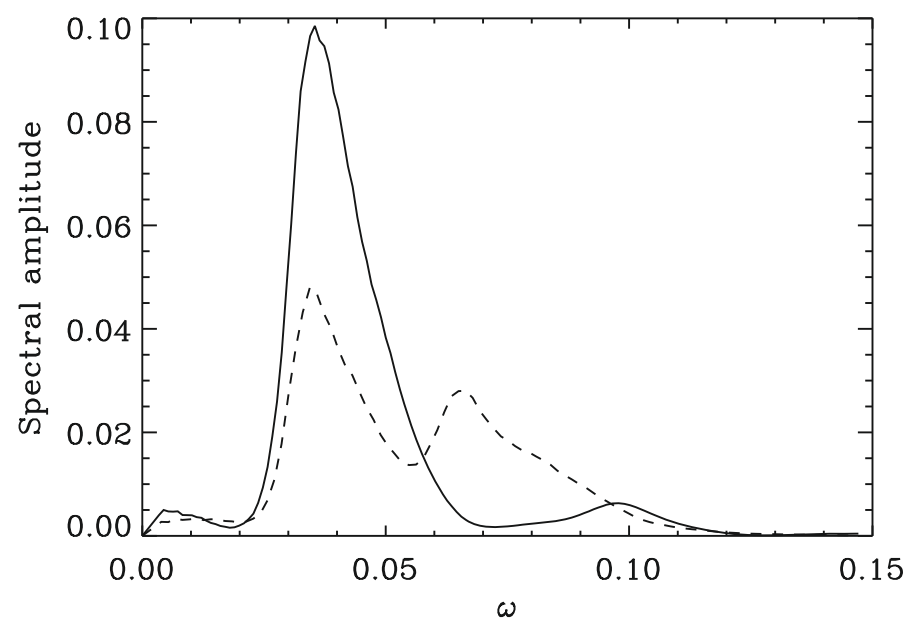

Fig. 13. Periodogram of the transverse velocity signal measured at the apex (solid line) and at $\theta=45$ degrees (dashed line) for an attack angle of 45 degrees (see Fig. 12).

of a higher harmonic. Figure 13 shows the periodogram of these transverse velocity signals. For the case of $\theta=45$ degrees there are two strong peaks, the lower frequency mode being the fundamental, also observed at the loop apex, and the higher frequency peak corresponding to the second harmonic.

Figure 14 shows the spectral amplitude of this second frequency as a function of the angle around the loop $\theta$ (see Fig. 2). The amplitude of the oscillation is zero at the footpoints and at the loop apex. There are two anti-nodes located between these three nodes, corresponding to a mode with a wavelength equal to the loop length, i.e. $N=2$.

The period of the harmonic is $P_{2}=95 \mathrm{~s}$. This gives us $P_{1} / 2 P_{2} \approx 0.9$, where the ratio is less than unity due to dispersion (see e.g Andries et al. 2005; McEwan et al. 2006).

Figure 15 shows this maximum spectral amplitude, measured at $\theta=45$, as a function of the attack angle. The lowest values of attack angle have been excluded since they efficiently excite the global mode (see Fig. 8) but inefficiently excite the second harmonic, and so the second harmonic peak is difficult to resolve. The second harmonic is most efficiently excited for attack angles $\alpha \approx 45$ degrees. This is consistent with the mode being a transverse oscillation (more efficiently excited by lower 


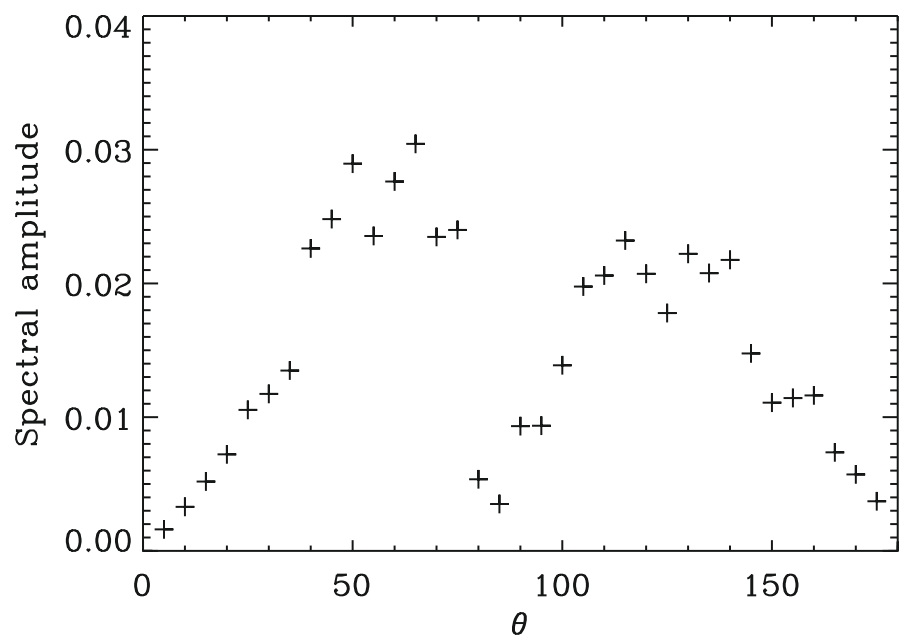

Fig. 14. The spectral amplitude of the second harmonic kink mode as a function of the angle around the loop $\theta$ for an attack angle of 45 degrees.

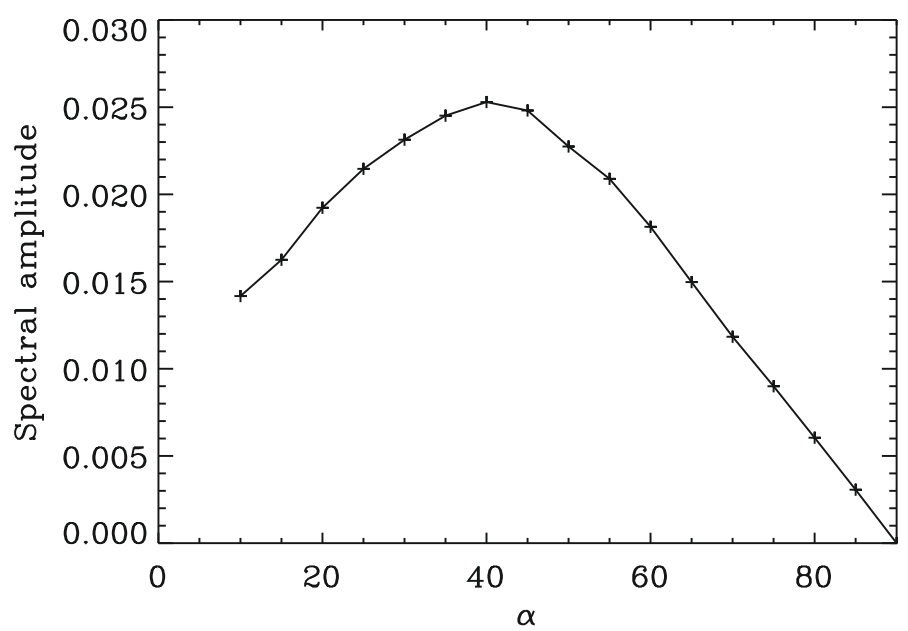

Fig. 15. The spectral amplitude as a function of the attack angle $\alpha$ for the second harmonic $(N=2)$ kink mode.

values of attack angle), and being anti-symmetric about the loop apex (requiring $\alpha \neq 0$ ).

We find that the fundamental kink mode is always more efficiently excited than the second harmonic. This is consistent with Terradas et al. (2007b) who consider the energy trapped in coronal loop eigenmodes when excited by an external perturbation. They found that the energy trapped in kink oscillations decreases with increasing $N$ and that this trend is insensitive to the details of the initial perturbation.

\subsection{Slow mode}

For small values of the attack angle, the impact of the pressure pulse is nearly perpendicular to the plane of the loop. However, for larger values of $\alpha$ the loop plasma is increasingly perturbed in the plane of the loop. This change in the impact angle leads to the excitation of an additional longitudinal mode, namely the slow mode. To correctly represent the longitudinal nature of this mode we use the velocity along the loop, $V_{\mathrm{s}}=V_{z} \cos \theta+V_{x} \sin \theta$ as the oscillation signal. Note that there is no mode conversion in our equilibrium as our loop is sufficiently far from $\beta=1$ (see Fig. 4) that the sound speed is never equal to the Alfvén speed.

Figure 16 shows the spectral amplitude of the slow mode as a function of the angle around the loop $\theta$ for an attack angle

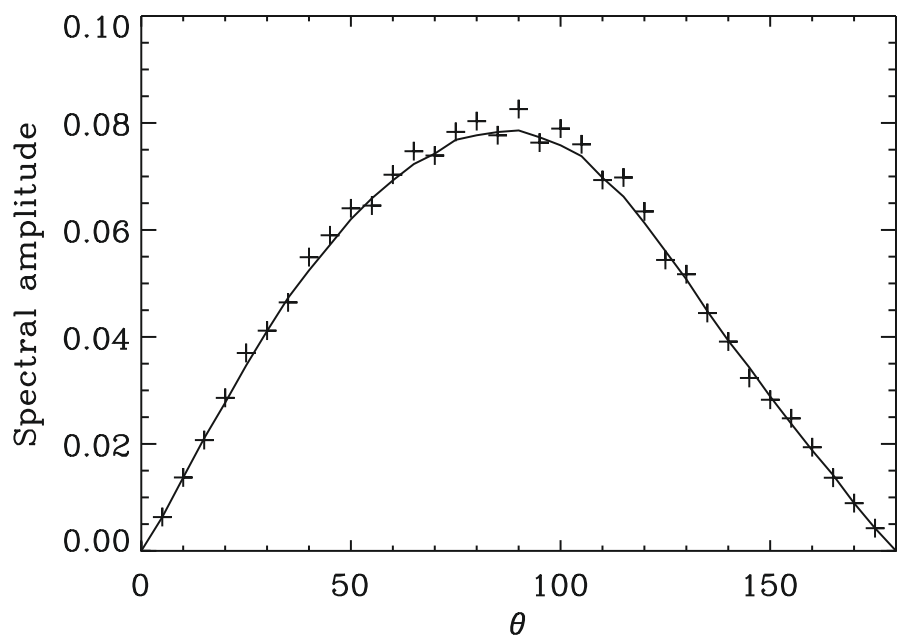

Fig. 16. The spectral amplitude of the slow mode as a function of the angle around the loop $\theta$ for an attack angle of 90 degrees.

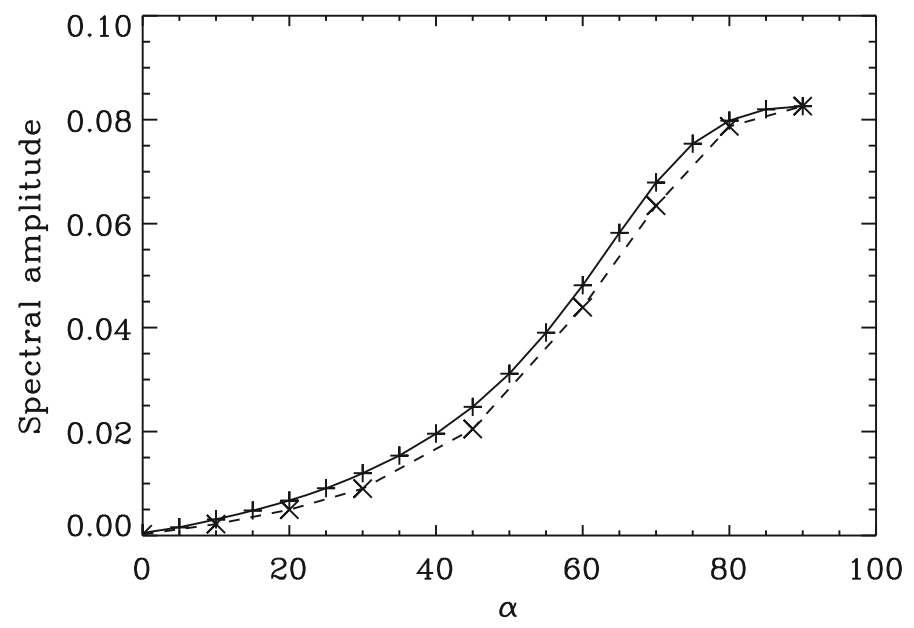

Fig. 17. The spectral amplitude of the slow mode as a function of the attack angle $\alpha$. The solid and dashed lines correspond to the Epstein and step density profiles, respectively.

of 90 degrees, i.e. the pressure pulse is actually situated in the plane of the loop. The amplitude of the oscillation is zero at the footpoints and has a single maximum at the apex, corresponding to a global mode with a wavelength of twice the loop length. Finally, Fig. 17 shows the spectral amplitude as a function of the attack angle $\alpha$. As expected, the excitation of the longitudinal mode is more efficient for larger attack angles and hence, the amplitude of the excited slow mode is larger for larger values of $\alpha$.

\section{Discussion}

We have studied the effect of the attack angle of a pressure pulse on a density-loaded coronal loop, embedded in an arcade magnetic field. These 3D simulations are a first step towards modelling coronal loop oscillations within a realistic active region environment. For small values of the attack angle, a global kink mode is excited, which is predominantly transverse in nature. As the value of the attack angle increases, the amplitude of the transverse oscillation decreases but an additional (longitudinal) slow mode is excited. In the most extreme case of a perpendicular attack angle (where the pressure pulse is situated in the plane of the loop), the transverse oscillation is no longer excited. We 
found the second harmonic kink mode was excited in addition to the fundamental mode for attack angles of about 45 degrees.

The slow mode was also present in 2D simulations by Selwa et al. (2006). These authors place a pulse beneath a coronal loop, which, as expected, excites fast (vertical) kink modes. However, if the pulse is situated asymmetrically, the fundamental slow mode is also found to be present. This study was extended by Selwa et al. (2007b) who compared a straight and curved geometry and conclude that the curvature facilitates the excitation of the slow mode.

One of the most striking properties of the observed transverse loop oscillations is the extremely rapid damping; in almost all observed examples, the oscillations are damped within a few periods (Schrijver et al. 2002; Aschwanden et al. 2002). Theoretically, the cause of this rapid damping is still not fully understood and several different mechanisms have been put forward as a potential explanation (Roberts 2004). In our numerical simulations, the transverse velocity signal at the loop apex is found to exhibit a similar rapid damping, with a signal quality generally less than two (see Fig. 11). As it is not the main focus of this paper, we did not investigate this rapid damping in detail but we can make a few general points. The most likely candidate to explain the rapid damping in our numerical simulations is the loop curvature. Damping due to lateral wave leakage in curved coronal loops has recently been the subject of a number of papers (e.g. Brady \& Arber 2005; Selwa et al. 2005, 2006, 2007a; Verwichte et al. 2006a-c; Diaz et al. 2006; Diaz 2006; Gruszecki et al. 2007, 2008). However, these studies are generally limited to $2 \mathrm{D}$ and hence are restricted to vertically polarised kink oscillations. Nevertheless, most of these studies similarly conclude that lateral wave leakage appears to be a very effective attenuation mechanism. In fact, Selwa et al. (2006, 2007a) as well as Verwichte et al. (2007) point out that (in 2D) the leakage is "too efficient" to explain the observed damping rates. Analysing 3D numerical simulations of coronal loop oscillations in a magnetic dipole, McLaughlin \& Ofman (2008) also find very rapid damping, which (using a comparison with a straight cylinder) they attribute to the loop curvature. However, due to the aspect ratio of our loop (a relatively "fat" loop with $a / L \approx 0.035$ ) the effect of curvature is probably stronger than it would be in realistic coronal loops. There is likely to be a numerical component to the rapid damping but doubling the numerical resolution did not result in an improvement in the signal quality. Hence, the majority of the damping must be caused by an actual physical damping mechanism. Since our equilibrium is inhomogeneous, resonant coupling of fast MHD waves and Alfvén waves can occur where the frequency of the global mode coincides with the local Alfvén frequency. However, we do not resolve the resonant layer and no significant dependence on density profile steepness was found and hence, damping by resonant absorption does not come into play. Although we tentatively suggest damping predominantly due to wave leakage (caused by the loop curvature), our simulations are not designed to investigate loop damping, as they do not have sufficient resolution to resolve other possible damping mechanisms (such as resonant absorption or phase mixing) and hence, no definite conclusions about the damping mechanism involved can be made here.

Clearly, the attack angle between the pressure pulse and the coronal loop affects the amplitude of the impulsively generated, transverse, kink mode in the coronal loop. For larger values of the attack angle (i.e. a pressure pulse travelling nearly parallel to the loop), the amplitude of the kink mode decreases and hence is much less likely to be observed. Therefore, the attack angle will contribute to the selectivity issue, possibly explaining why only some loops are seen to oscillate. However, very little information on the attack angle exists from observations and in most cases, projection effects make it very difficult to accurately determine the value of the attack angle. Observations by STEREO/EUVI now allow for the 3D reconstruction of coronal loops and hence should give a much clearer picture of the local active region geometry (Aschwanden 2009). So far, no flare-induced coronal loop oscillations have been observed by EUVI but hopefully increasing solar activity in the future will lead to actual observations of transverse loop oscillations with STEREO/EUVI. However, the attack angle can not solely be responsible for the selectivity in oscillating loops, as in some instances, neighbouring loops (for which the attack angle would be nearly identical) do not all appear to be excited.

An unexpected feature of our numerical simulation is that the excited kink mode period is considerably smaller than theoretically expected (165 s versus $260 \mathrm{~s}$ ). This was noted previously by Miyagoshi et al. (2004) who found that for a curved geometry, the period appears to scale as $P \sim \rho_{0}^{0.33}$ rather than the expected $P \sim \rho_{0}^{0.5}$, where $\rho_{0}$ is the density inside the coronal loop. Using this scaling in our study, the theoretically predicted period (for a straight geometry) of $260 \mathrm{~s}$ would reduce to about $175 \mathrm{~s}$, which is close to the period obtained from the numerical simulations. Comparing Fig. 8c (curved loop) and Fig. 9c (straight loop) of McLaughlin \& Ofman (2008), this scaling also seems to be present (although it was not investigated in detail by these authors). This strong modification of the period due to the curvature appears to contradict the result of Van Doorsselaere et al. (2004), who found that the periods of oscillations were only marginally affected by the curvature. However, their analysis is valid for thin coronal loops, whereas our numerical loop is relatively thick. This modified periodicity is a consequence of the combination of curvature, a relatively large density contrast $\left(\rho_{0} / \rho_{\mathrm{e}}=10\right)$ and aspect ratio $(a / L \approx 0.035)$. We plan to investigate this modification in more detail in a future parameter study. Realistic coronal loops are expected to have a smaller aspect ratio than the one we used in our model (due to restriction in numerical resolution) and hence, it is possible that this reduction in period would not occur in oscillating loops strands in the solar corona. On the other hand, several studies have recently pointed out that the modes of oscillation do not appear to be sensitive to fine-structure but can behave as a collective, global mode (e.g. Diaz et al. 2005; Gruszecki et al. 2006; Luna et al. 2006; Pascoe et al. 2007) and hence, oscillating loop strands situated close together could be affected by this modification in the periodicity.

\section{Conclusions}

In this paper, we have investigated the interaction of a fast MHD wave, generated by impulsive energy deposition (pressure pulse), with a 3D coronal loop. As the fast wave passes the coronal loop, modelled as a density enhancement within a magnetic arcade structure, a transverse loop mode is excited. Qualitatively, our 3D simulation of the transverse, global kink mode are consistent with theoretical predictions. The excited kink modes are global modes, with a single, dominant period and maximum amplitudes at the loop apex. The period of the transverse kink mode is independent of the attack angle but is substantially smaller than theoretically predicted. This implies a phase speed close to the external Alfvén speed, rather than the internal Alfvén speed. This reduction in the period is due to the combination of curvature, a large aspect ratio and high density contrast and will be investigated in detail in future work by comparison with eigenmode calculations for our equilibrium. Comparing a modified 
(smooth) Epstein profile with a step profile density enhancement, we find that the excitation and damping only weakly depend on the density profile steepness. The global, transverse kink mode is efficiently excited for a relatively wide range of attack angles ( $\alpha<60$ degrees) and hence the attack angle can at best only partially account for the observed selectivity of the kink mode. For the larger values of $\alpha$, the global (longitudinal) slow mode is excited. We also see that for $\alpha \approx 45$ degrees the second harmonic kink mode is generated.

Many open questions remain and several aspects of the model can be developed further. For example, a more typical magnetic field structure for the solar corona (such as a dipole) could be considered. We have used a relatively high value of the coronal plasma beta $(\beta \sim 0.1$ at the loop apex) to suppress the effect of the ponderomotive force. As shown by Terradas \& Ofman (2004), for lower values of beta $(\beta \sim 0.01)$, the ponderomotive force will have to be taken into account, or one could consider loops in which beta varies as a function of height. Observed transverse, flare-induced, kink mode oscillations manifest themselves by a clear, oscillatory displacement of the coronal loop(s). In our current model, there is a transverse velocity signal at the apex but the actual loop displacement is small and would be below the resolution of current imaging instruments. Hence, larger amplitudes will be considered (see De Moortel \& Pascoe 2009) to investigate how the actual loop displacements compare to observed loop oscillations.

Acknowledgements. D.J.P. and J.A.M. acknowledge financial support from STFC and the Leverhulme Trust, respectively. I.D.M. acknowledges support of a Royal Society University Research Fellowship. The computational work for this paper was carried out on the joint STFC and SFC (SRIF) funded cluster at the University of St. Andrews (Scotland, UK). The authors would like to thank Prof. P. J. Cargill for insightful discussion, and the referee for helpful comments.

\section{References}

Andries, J., Arregui, I., \& Goossens, M. 2005, ApJ, 624, L57

Arber, T. D., Longbottom, A. W., Gerrard, C. L., et al. 2001, JCP, 171, 151

Arregui, I., Andries, J., Van Doorsselaere, T., Goossens, M., \& Poedts, S. 2007, A\&A, 463, 333

Aschwanden, M. J. 2009, Space Sci. Rev., 23

Aschwanden, M. J., Fletcher, L., Schrijver, C. J., et al. 1999, ApJ, 520, 880

Aschwanden, M. J., De Pontieu, B., Schrijver, C. J., et al. 2002, Sol. Phys., 206, 99

Ballai, I. 2007, Sol. Phys., 246, 177

Banerjee, D., Erdélyi, R., Oliver, R., et al. 2007, Sol. Phys., 246, 3

Brady, C. S., \& Arber, T. D. 2005, A\&A, 438, 733

Brady, C. S., Verwichte, E., \& Arber, T. D. 2006, A\&A, 449, 389

Cally, P. S. 1986, Sol. Phys., 103, 277

Cally, P. S. 2003, Sol. Phys., 217, 95

Del Zanna, L., Schaekens, E., \& Velli, M. 2005, A\&A, 431, 1095

De Moortel, I. 2005, Phil. Trans. R. Soc. London, Ser. A, 363, 2743

De Moortel, I., \& Brady, C. S. 2007, ApJ, 664, 1210

De Moortel, I., \& Pascoe, D. J. 2009, ApJ, 699, L72

Diaz, A. J. 2006, A\&A, 456, 737

Diaz, A. J., Oliver, R., \& Ballester, J. L. 2005, A\&A, 440, 1167
Diaz, A. J., Zaqarashvili, T., \& Roberts, B. 2006, A\&A, 455, 709

Edwin, P. M., \& Roberts, B. 1983, Sol. Phys., 88, 179

Erdélyi, R., \& Verth, G. 2007, A\&A, 462, 743

Gruszecki, M., Murawski, K., Selwa, M., \& Ofman, L. 2006, A\&A, 460, 887

Gruszecki, M., Murawski, K., Solanki, S. K., \& Ofman, L. 2007, A\&A, 469, 1117

Gruszecki, M., Murawski, K., \& McLaughlin, J. A. 2008, A\&A, 489, 413

Heyvaerts, J., \& Priest, E. R. 1983, ApJ, 117, 220

Luna, M., Terradas, J., Oliver, R., \& Ballester, J. L. 2006, A\&A, 457, 1071

McEwan, M. P., Donnelly, G. R., Díaz, A. J., \& Roberts, B. 2006, A\&A, 460, 893

McLaughlin, J. A., \& Ofman, L. 2008, ApJ, 682, 1338

Miyaghoshi, T., Yokoyama, T., \& Shimojo, M. 2004, PASJ, 56, 207

Nakariakov, V. M. 2007, Adv. Space Res., 39, 1804

Nakariakov, V. M., \& Ofman, L. 2001, A\&A, 372, L53

Nakariakov, V. M., \& Roberts, B. 1995, Sol. Phys., 159, 399

Nakariakov, V. M., \& Verwichte, E. 2005, Living Reviews in Solar Physics, 2, http: //www . livingreviews . org/lrsp-2005-3 (cited August 2007)

Nakariakov, N. M., Ofman, L., DeLuca, E., Roberts, B., \& Davila, J. M. 1999, Sci, 285, 862

Ofman, L. 2005, Adv. Space Res., 36, 1572

Ofman, L. 2007, ApJ, 655, 1134

Ofman, L. 2009, ApJ, 694, 502

Ofman, L., \& Aschwanden, M. J. 2002, ApJ, 576, L153

Ofman, L., \& Thompson, B. J. 2002, ApJ, 574, 440

Ofman, L., \& Wang, T. J. 2008, A\&A, 482, L9

Pascoe, D. J., Nakariakov, V. M., \& Arber, T. D. 2007, Sol. Phys., 246, 165

Pascoe, D. J., Nakariakov, V. M., Arber, T. D., et al. 2009, A\&A, 494, 1119

Roberts, B. 2000, Sol. Phys., 193, 139

Roberts, B. 2004, SOHO 13 Proceedings, ESA SP-547, P1

Roberts, B., Edwin, P. M., \& Benz, A. O. 1983, Nature, 305, 688

Roberts, B., Edwin, P. M., \& Benz, A. O. 1984, ApJ, 279, 857

Ruderman, M. S., \& Roberts, B. 2002, ApJ, 577, 475

Schrijver, C. J., Aschwanden, M. J., \& Title, A. 2002, Sol. Phys., 206, 69

Selwa, M., Murawski, K., Solanki, S. K., Wang, T. J., \& Tóth, G. 2005, A\&A, 440,385

Selwa, M., Solanki, S. K., Murawski, K., Wang, T. J., \& Shumlak, U. 2006, A\&A, 454, 653

Selwa, M., Murawski, K., Solanki, S. K., et al. 2007a, A\&A, 462, 1127

Selwa, M., Ofman, L., \& Murawski, K. 2007b, ApJ, 668, L83

Taroyan, Y., Erdélyi, R., Wang, T. J., \& Bradshaw, S. J. 2007, ApJ, 659, L173

Terradas, J., \& Ofman, L. 2004, ApJ, 610, 523

Terradas, J., Oliver, R., \& Ballester, J. L. 2005a, ApJ, 618, L149

Terradas, J., Oliver, R., \& Ballester, J. L. 2005b, A\&A, 441, 371

Terradas, J., Oliver, R., \& Ballester, J. L. 2006a, Phil. Trans. R. Soc. London, Ser. A, 364, 547

Terradas, J., Oliver, R., \& Ballester, J. L. 2006b, ApJ, 650, L91

Terradas, J., Andries, J., \& Goossens, M. 2007a, Sol. Phys., 246, 231

Terradas, J., Andries, J., \& Goossens, M. 2007b, A\&A, 469, 1135

Uchida, Y. 1970, PASJ, 22, 341

Van Doorsselaere, T., Debosscher, A., Andries, J., et al. 2004, A\&A, 424, 1065

Van Doorsselaere, T., Nakariakov, V. M., \& Verwichte, E. 2007, A\&A, 473, 959

Van Doorsselaere, T., Nakariakov, V. M., Young, P. R., et al. 2008, A\&A, 487, L17

Verth, G., Van Doorsselaere, T., Erdélyi, R., et al. 2007, A\&A, 475, 341

Verwichte, E., Nakariakov, V. M., Ofman, L., et al. 2004, Sol. Phys., 223, 77

Verwichte, E., Foullon, C., \& Nakariakov, V. M. 2006a, A\&A, 446, 1139

Verwichte, E., Foullon, C., \& Nakariakov, V. M. 2006b, A\&A, 449, 769

Verwichte, E., Foullon, C., \& Nakariakov, V. M. 2006c, A\&A, 452, 615

Wang, T. J., \& Solanki, S. K. 2004, A\&A, 421, L33

Wang, T. J., Innes, D. E., \& Qiu, J. 2007, ApJ, 656, 598

Wang, T. J., Solanki, S. K., \& Selwa, M. 2008, A\&A, 489, 1307 